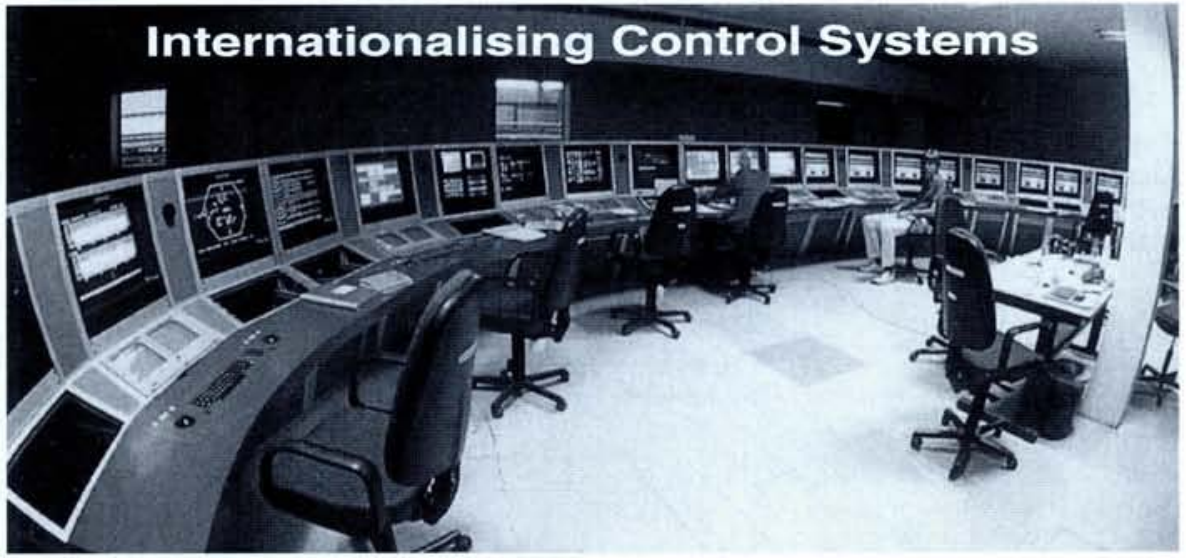

The control room of the HERA accelerator, DESY, Hamburg.

Within 10 years from now, the manpower required for writing applications software for large experimental physics control systems will exceed a few hundred to a few thousand man-years per system. In order to handle this huge effort one must establish standard models, adopt adequate computer software engineering methods and tools (among which

the object-oriented appear quite promising), set up of standard protocols, and construct application environments via world-wide collaboration. Hence the importance of ICALEPCS, the biennial International Confer-' ence on Accelerator and Large Experimental Physics Control Systems, that covers the systems used to pilot the wide variety of large

\section{Current Trends in Data Acquisition and Control of Accelerators}

A conference organized in Calcutta, India, in November 1991 by India's Department of Atomic Energy (DAE) in collaboration with the EPS controls systems group (EPCS) showed how the group can help developing countries learn how to design and build modern controls systems for major experiments. About 80 participants from Indian labs met with specialists from US and European centres to discuss topics ranging from detailed standards and methods for both hardware and software to the management of accelerator

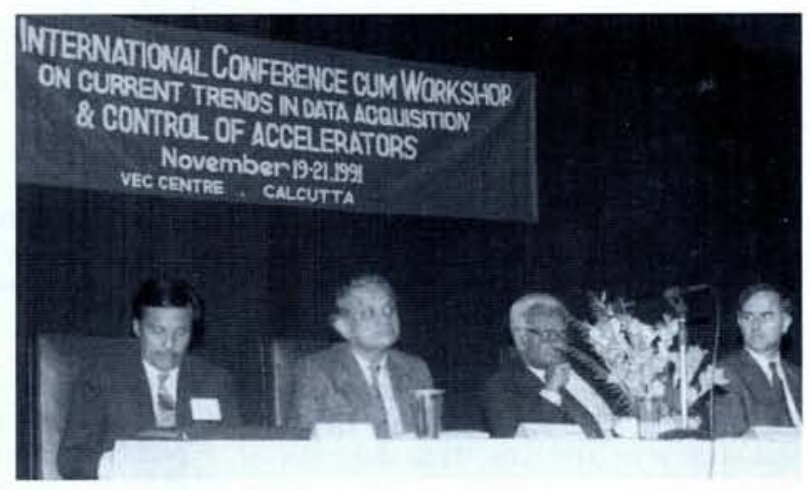

From the left, S. Dasgupta, Dr. B. Sinha, Dr. B.R. Raychowdhury, Vice-Chancellor, Calcutta University, and A. Daneels, Chairman EPCS, at the opening of the Calcutta data acquisition and controls conference. facilities. The initiative mainly came from $\mathrm{Dr}$. B. Sinha, the Director of Calcutta's Variable Energy Cyclotron Centre (VECC), where a control system is needed for a superconducting cyclotron with the same name which is under construction. On a larger scale, the DAE has entered a 50 MSUS cooperation programme with the SSC collider in Texas.

The suitability and limitations of portable blem of communications in distributed control systems, were stressed as India generally adopts these approaches. Workshops on toolkits for application software and on protocols for instrument and power supply controls were a great success and gave excellent overviews of control standards for accelerators.

S. Dasgupta, VECC, Calcutta computers for control systems, and the pro-

Université de Nancy-I

Département de Physique

\section{AVIS DE VACANCE DE POSTE}

L'Université de Nancy-l est susceptible de recruter au 01.10.1993 un PROFESSEUR Spécialité PHYSIQUE DU SOLIDE.

Le candidat aura une expérience de l'étude de la physique des surfaces et des interfaces, de leurs propriétés magnétiques, et des spectroscopies liées aux processus d'interaction rayonnement-matière. II animera une équipe de recherche dans ce domaine et sera appelé à prendre la responsabilité du service commun de photoémission existant à l'Université.

Le candidat exercera son activité d'enseignement à la Faculté des Sciences, en physique générale (DEUG-Licence) et en physique du solide (Maîtrise-DEA).

Pour pouvoir être recruté à titre permanent, le candidat devra être inscrit sur la liste de qualification aux fonctions de professeur.

Renseignements complémentaires auprès du responsable du Département de Physique, Faculté des Sciences, BP 239, F-54506 Vandoeuvre-les-Nancy Cédex. instruments found in physics. These range from high-energy detectors and fusion reactors to telescopes, and not just high-energy particle accelerators.

The series started as workshops in the USA in 1985 and matured under the EPS Interdivisional Group on Experimental Physics Control Systems (EPCS) into biennial international conferences held in Villars-surOllon, Switzerland (1987), Vancouver, Canada, (1989), and Tsukuba, Japan (1991).

The five general trends were clearly identified at the Tsukuba conference. The idea behind a standard model of control systems is to distribute a control system on three layers, where each layer is composed of hardware and software based on international or de facto international standards. While recent progress in computer technology enables us to propose this kind of model, it is premature to say that it exists at present. Nonetheless, trying to establish it is a move in the right direction.

Several laboratories are joining efforts to introduce modern computer-aided software engineering (CASE) to improve software efficiency. Techniques and supporting tools are being evaluated, and although the more classical structured analysis, structured design, (SASD) method seems to be gaining ground, there exist today a few control systems based on object-oriented concepts (e.g. those for AGS Booster, in the USA, and for the ESRF, Grenoble). One cannot yet judge to what extent CASE-tools reduce manpower requirements and improve the quality of control systems. Estimating the effectiveness of object-orientated methodology will be possible in a few years when many control systems will have been developed using the approach.

Standard protocols represent ways to model accelerators from the viewpoint of controls and European collaboration led by the EPCS is making this methodology more widely accepted.

Increased exploitation of open systems, standardization, object-oriented concepts, and standard protocols will make it possible to define specifications and to construct a generic applications environment through world-wide collaboration. Indeed, we can reasonably hope that initiatives to create such an environment will become full-fledged in a few years.

Approximately 220 participants attended ICALEPCS ' 91 , including 140 from outside Japan, of which 50 were specialists from other parts of Asia, and from the former Soviet Union, who had not participated before. There were 150 contributed papers covering everything from status reports to details of programmes. The next ICALEPCS on 1822 October 1993 at the Hahn-Meitner Institute, Berlin, has already attracted sponsorship equivalent to the 1991 event when EPS IEEE, NPSS, and The Physical Society of Japan were sponsors. ICALEPCS '93 promises an even greater level of interest with sessions covering system architecture, CASEtools, commercial products, man-machine interfaces, modelling, feedback control, software sharing, and industrial cooperation. An industrial exhibition is planned and abstracts are due by 31 March 1993. Detailed information is available from: Dr. W. Busse, HahnMeitner-Institut, Postfach 3901 28, W-1000 Berlin 39 (tel./fax: +49-30-80 092330 / 8009 20 97; email: busse@vax.hmi.dbp.de). 